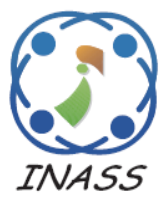

\title{
Detection of Overlapping Teeth on Dental Panoramic Radiograph
}

\author{
Agus Zainal Arifin ${ }^{1 *}$ \\ Safri Adam ${ }^{1}$ \\ Avin Maulana Mohammad ${ }^{1}$ \\ Fatoni Anggris $^{1} \quad$ Rarasmaya Indraswari $^{1} \quad$ Dini Adni Navastara ${ }^{1}$ \\ ${ }^{I}$ Department of Informatics, Faculty of Information and Communication Technology, \\ Institut Teknologi Sepuluh Nopember (ITS), Surabaya, Indonesia \\ *Corresponding author’s Email: agusza@cs.its.ac.id
}

\begin{abstract}
Segmentation of single tooth in dental panoramic images is an important process to extract its features and information. However, it might be challenging when the segmentation process faces an overlapping teeth image. In this research, we introduce a new strategy for detecting overlapping area on dental panoramic radiographs automatically. This research proposes automatic thresholding to obtain marking points for the overlapping area and an automatic selection of overlapping area candidates by using the area orientation and the similarity of neighborhood intensity. The experimental results on 44 images show that our proposed strategy can detect overlapping teeth on the dental panoramic radiograph with accuracy, sensitivity, and specificity of $75 \%, 66.67 \%$, and $85 \%$, respectively. The evaluation conducted on 24 overlapping teeth images shows that the segmentation results of overlapping teeth area have an average misclassification error of $0.31 \%$.
\end{abstract}

Keywords: Dental panoramic radiograph, Overlapping teeth detection, Segmentation.

\section{Introduction}

Dental panoramic radiograph image provides many benefits in the odontology field. By using the image, dentists can analyze and diagnose some part of the tooth that is not directly visible. An image contains useful information that we can understand by extracting the features from the image. An important step to extract information from the image is by using image segmentation [1]. Image segmentation is conducted to distinguish the object (foreground) and the non-object (background) in the image [2]. The object in dental panoramic radiograph usually is the tooth. Image segmentation process with various algorithms, such as in [2-5], achieve a good result for segmentation of single tooth image. However, it might be challenging when the segmentation process faces an overlapping teeth image. In the case of dental age assessment on panoramic radiograph images, one of the difficulties in the assessment process is the presence of overlapping teeth [6]. Overlapping objects make the interpretation process more complicated. This problem makes the interpretation process take a lot of time and require highly trained personnel to avoid high error rate [7]. In image segmentation, the overlapping objects could cause inaccurate segmentation result because there are parts of an object that shadow other objects, therefore produces a wrong contour [8].

Overlapping teeth are common in dental panoramic radiographs. One of the causes is the process of natural changes of primary teeth to permanent teeth that occur in people under 17 years old. In this process, the permanent teeth that grow after the primary teeth causing overlap between those teeth [9]. Another cause of overlapping teeth caused is the abnormal growth of the teeth. Image segmentation methods will segment the overlapping teeth into one object. To segment and separate two overlapping teeth, it is necessary to detect the overlapping area. The unique characteristic of the overlapping area on dental panoramic radiographs is it usually has higher grayscale intensity than others. However, it is inadequate to just depend on the grayscale intensity for detecting the overlapping 
area because there are other areas in the dental panoramic radiograph that has high intensity, such as the enamel.

Therefore, in this research, we introduce a new strategy for detecting overlapping area on dental panoramic radiographs automatically. This research proposes automatic thresholding to obtain marking points for the overlapping area and an automatic selection of overlapping area candidates by using the area orientation and the similarity of neighborhood intensity. The proposed strategy utilizes information from dental panoramic images in the automatic marking process, in which overlapping areas usually have high grayscale intensity values. Based on the obtained marking points, several regions are selected as the candidate of the overlapping area. The information of area orientation and the similarity of neighborhood intensity is used to eliminate other high-intensity areas, such the enamel. The proposed method is fully automatic and can be applied to detect overlapping teeth in various dental panoramic radiographs. The detection of overlapping teeth area is important because it will affect the segmentation of a single tooth in dental panoramic images. The segmentation of single tooth further can be used for dental surgery and medical forensic.

This paper is organized as follows. Section 2 discusses the related works about the detection of overlapping objects in general. Section 3 presents the proposed methodology for the detection of overlapping teeth on dental panoramic radiograph. Section 4 describes the experiments that have been performed and presents their results. The conclusion and the future work of this research are presented in Section 5 .

\section{Related works}

Image segmentation methods will segment two overlapping teeth into one object. This is a problem when the expected result of the segmentation process is a single tooth. Research that uses panoramic radiographic images states the presence of overlapping teeth is a major challenge in getting a single tooth for the next process $[10,11]$. There are several researches about image segmentations with overlapping objects. Wang, et al. proposed a segmentation method of overlapping plant leaves by using the Chan-Vese method and the Sobel operator [8]. This method gives good performance to separate objects in natural images. However, natural images have different characteristics with medical images, such as X-ray images. One of them is the overlapping areas on $\mathrm{X}$-ray images is marked by the changes in grayscale intensity (superimposition). Therefore this method is not suitable for detecting the overlapping teeth on dental panoramic radiographs.

Mahyari \& Dansereau (2017) conducted the segmentation of object containing translucent overlapped regions in synthetic images using random walker algorithm [12]. In that process, the multi-layer graph is made from 2-dimension images containing translucent overlapping area and generate a Laplacian matrix based on a multi-layer graph. The Laplacian matrix provides a small amount of user-defined label. This method gives accuracy on separating overlapping translucent objects. The translucent objects give similar characteristic with the dental panoramic radiograph, which is intensity changes in overlapping areas. However, the need for user-defined label for the segmentation process can be exhaustive to apply on large amount of dataset.

Overlapping object image segmentation has also been done by Riana, et al. (2017) on pap smear images [13]. The research focused on the segmentation of overlapping cytoplasmic objects and nucleus detection. To get the overlapping area, the labeling process is performed to mark the object area and region properties, therefore a bounding box that ensures the object is part of the overlap can be obtained. The overlapping area on pap smear images can be detected by using the presence of nuclei as the sign of a cell, therefore the dividing line between two overlapping objects can be determined. This cannot be applied to the dental panoramic radiographs which do not have a clear marker between two teeth.

Yadollahi, et al. (2005) separates overlapping dental objects using the normal vector principle to detect the intersection of adjacent teeth edges in the gypsum image of the dental arch component [14]. The overlapping object is defined by teeth that are attached to each other on the tooth gypsum image. However, this method is applied to the images that show the cross-section of the teeth from the top, where the overlapping area is very small and the structure of two overlapping teeth is clearly visible because it is circular. This cannot be applied to the dental panoramic radiographs which show the crosssection of the teeth from the front, where the size of the overlapping area vary and the structure of the two overlapping teeth cannot be clearly distinguished.

\section{Material and methods}

The data used in this research is the region of 


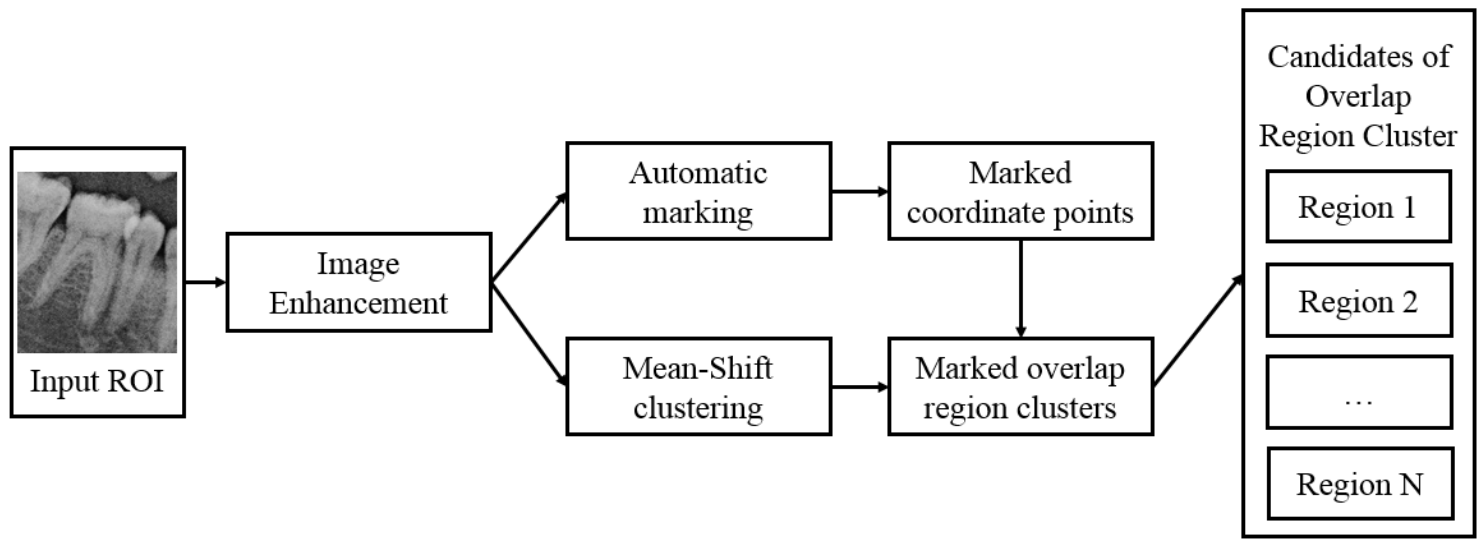

Figure. 1 Proposed methodology

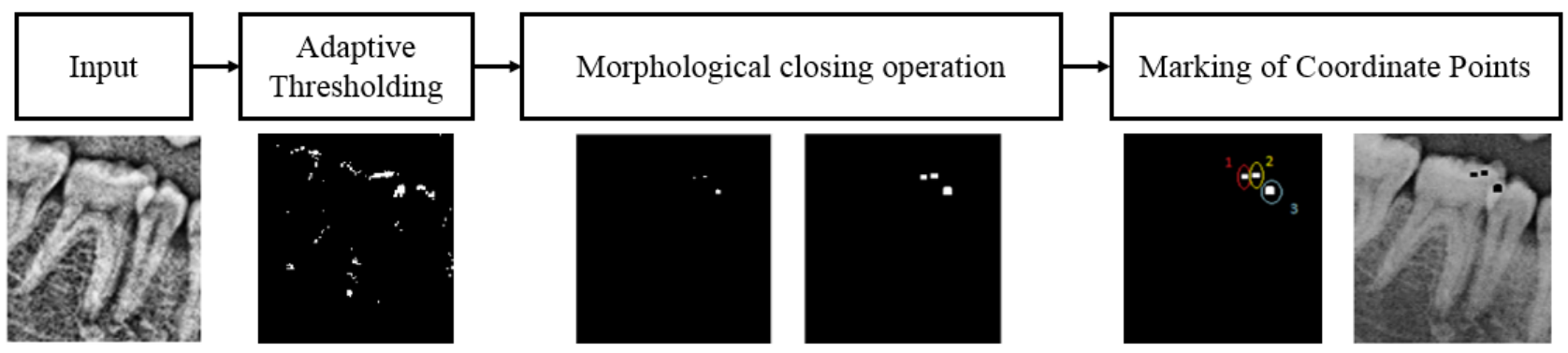

Figure. 2 Automatic marking of coordinate points

interest (ROI) from dental panoramic radiograph images that contains the overlapping teeth. To obtain the ROI, observations are conducted on several dental panoramic radiograph images with size 1976 pixels $\times 976$ pixels. Observations are conducted by looking for overlapping tooth objects and cropping process is done manually over two overlapping teeth.

In this research, several processes are conducted to detect the overlapping teeth area. Those processes are image enhancement, automatic marking of coordinate points, mean-shift image clustering, and selection of overlapping object candidates. The methodology of the overlapping detection system can be seen at Fig. 1 .

\subsection{Image enhancement}

Contrast Limited Adaptive Histogram Equalization (CLAHE) method is used in the image enhancement process. CLAHE is one of image enhancement techniques that have been widely used in many applications of image processing. The purpose of CLAHE is to enhance low-contrast images, as a pre-processing of the input image. The CLAHE algorithm divides the image into regions, then optimize the contrast of each region. CLAHE was developed to overcome the noise or artifact problem that emerges on Adaptive Histogram Equalization (AHE), by limiting contrast enhancement especially in the homogeneous area [15].

\subsection{Automatic marking of coordinate points}

After the contrast of the input image is enhanced, the next process is the automatic marking of coordinate points of the overlapping region candidates. By using the enhanced ROI image as the input, there are several steps that need to be done to obtain the coordinate points of the overlapping region candidates, which are image thresholding, morphological operation for refinement of segmentation result, and automatic marking of coordinate points. Those steps are shown in Fig. 2.

Most of medical image segmentation methods are based on pixel intensity. Segmentation method based on pixel intensity is the classification of pixel intensity that uses a statistical algorithm to set the label of pixels in an image [16]. One of the common methods for segmentation is Otsu's thresholding method [17]. The basic principle of the Otsu's thresholding is to get obtain the best separation threshold between the intensity of object and background pixels by using the statistical inter-class variance. Let $\sigma_{B}^{2}$ be the inter-class variance between class $O$ (background) and class 1 (object). The Otsu's thresholding method search the value of graylevel $k$ that gives the maximus inter-class variance by calculating the probability of class 
occurrence $\omega$ and the average graylevel of the class $\mu$ according to Eq. (1).

$$
\sigma_{B}^{2}(k)=\omega_{0} \omega_{1}\left(\mu_{1}-\mu_{0}\right)^{2}
$$

In dental panoramic radiograph images, the segmentation process is globally desired to distinguish the tooth object and non-object. In this research, we introduce a new method to get the overlapping area of teeth in the dental panoramic radiograph. Based on the observation result, it was found that overlapping teeth have a higher grayscale intensity than the tooth object itself. To obtain the overlapping teeth, adaptive thresholding is done. First, the histogram of grayscale intensity, with range $0-255$, of the enhanced ROI image is constructed. Based on observations, the grayscale intensity of the overlapping areas is above 192. Therefore, we split the $x$-axis of the histogram into four regions and find the threshold of the overlapping area on the $H_{4}$ region on the histogram according to Fig. 3 .

After splitting the $x$-axes in the histogram, Otsu's thresholding is used to get a threshold on the $\mathrm{H}_{4}$ region on the histogram. The obtained threshold is the best grayscale intensity to separate the object, which is the overlapping areas with grayscale values between 193-255, and the non-object. This strategy is robust for all dental panoramic radiograph images to segment overlapping object. However, because there are other areas in the dental panoramic radiograph that have grayscale intensity above 192, such as the enamel and the small part of dental tissues, those areas are also segmented. Therefore, further process, which is the morphological closing operation, is needed to eliminate the irrelevant small parts of the thresholding result.

Morphological operations on image processing are non-linear operations that are related to the shape or morphology of features in an image. Morphological operations only depend on the relative order of pixel values, not on their numerical values. Therefore they are very suitable for binary image processing [18]. The basic morphological operations are erosion, dilation, opening, and closing.

In this research, morphological closing with a disc structural element is used. Morphological closing is used to eliminate the small-size pixels on the result of the thresholding process. In determining

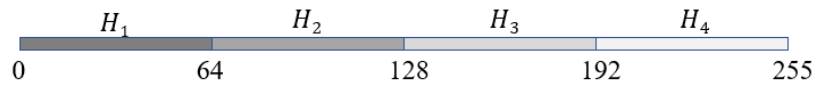

Figure. 3 Partition of the histogram region the size and shape of the structural element, we refer to the previous research conducted by Wisaeng (2019) that used disc structural element with size 7 because it is computationally efficient [19]. The result of this process is then saved for the next process in selecting the overlapping area candidates.

\subsection{Mean-shift image clustering}

The mean-shift algorithm is a non-parametric feature-based or space-based analysis technique [20]. This algorithm can be applied to analyze cluster in image processing [21]. Given $n$ number of data points $X=\left\{x_{1}, x_{2}, x_{3}, \ldots, x_{n}\right\}$ and symmetrical kernel $K$, mean-shift vector is defined as $P(x)$ as in Eq. (2). To obtain the initial segmentation clusters of the image we use the mean-shift segmentation software made by the Edison System [22]. The input enhanced ROI is divided into several regions based on the probability density gradient function. The results of the initial segmentation using a mean-shift algorithm are better than other image clustering methods, because they consider spatial information and the shape of the object in the image [23]. The region clusters which contain the mark from the automatic marking process are selected as the candidate of the overlapping teeth area.

$$
P(x)=\frac{1}{n} \sum_{i=1}^{n} K(x-x i)
$$

\subsection{Selection of overlapping object candidates}

The candidates of the overlapping object are identified by the two steps as we propose, which are the non-overlapping object elimination by using the object orientation and the overlapping object detection by using the neighborhood similarity. In the non-overlapping object elimination, object orientation is used as a guide in identifying the overlapping and non-overlapping object, where the orientation of each overlapping object cluster is identified. A non-overlapping teeth area, such as the enamel, usually have a horizontal orientation. Let a line parallel to the $\mathrm{x}$-axis has an angle of $0^{\circ}$ and the calculation of the degree of slope $\theta$ is according to counter-clockwise rotation as in Fig. 4. A cluster is classified as an overlapping object if its main orientation is vertical with the degree of slope $45^{\circ}<$ $\theta<135^{\circ}$ or $225^{\circ}<\theta<31$. A cluster is classified as a non-overlapping object if its main orientation is horizontal with degree of slope $0^{\circ} \leq \theta \leq 45^{\circ}$ or $135^{\circ} \leq \theta \leq 225^{\circ}$ or $315^{\circ} \leq \theta \leq 360$. The clusters that are classified as an overlapping object will be further processed in the next step. 


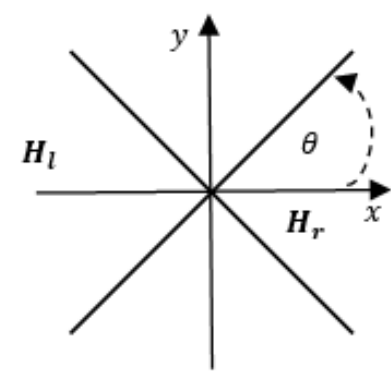

Figure. 4 Determination of neighbors relative to $c_{i}$

Let the overlapping region candidates denoted by $C=\left\{c_{1}, c_{2}, c_{3}, \ldots, c_{n}\right\}$ where $n$ is the number of regions that are not eliminated after the first step. The selection of overlap candidates is carried out by comparing the average gray-level intensity $\mu(G)$ of the neighboring regions of each overlap candidates. The average intensity of a region that consists of $m$ number of pixels with gray-level intensity $x$ and probability of each gray-level $h(x)$ is defined by Eq. (3). The average intensity is calculated on the cluster of overlap candidates and its neighboring clusters.

$$
\mu(G)=\frac{\sum_{j=1}^{m} x_{j} h\left(x_{j}\right)}{\sum_{j=1}^{m} h\left(x_{j}\right)}, x_{j} \in G
$$

An overlap candidate will be selected as an overlapping teeth area if the intensity of its right and left neighboring cluster was similar, but have lower intensity than the overlap candidate cluster $c_{i}$. This is because the right and left area of an overlapping teeth area are the teeth, which have similar intensity. Let the coordinate of center mass of each cluster is expressed as $O_{G}=\left(x_{G}, y_{G}\right)$. Neighboring clusters of the overlap candidate cluster $c_{i}$ is notated as $N_{i}=$ $\left\{n_{i 1}, n_{i 2}, \ldots, n_{i k}\right\}$ where $k$ is the number of clusters that bordering cluster $c_{i}$. According to Fig. 4, the left neighboring cluster of cluster $c_{i}\left(H_{l}\right)$ is the neighboring cluster that located at an angle of $135^{\circ} \leq \theta \leq 225^{\circ}$ from the cluster $c_{i}$. The right neighboring cluster of cluster $c_{i}\left(H_{r}\right)$ is the neighboring cluster that located at an angle of $0^{\circ} \leq$ $\theta \leq 45^{\circ}$ or $315^{\circ} \leq \theta \leq 360^{\circ}$ from the cluster $c_{i}$. The average intensity of the right and left neighboring cluster is similar if their difference is below certain threshold $\varepsilon$. Cluster $c_{i}$ is declared as an overlapping teeth area if it meets the criteria in Eq. (4).

$$
\left|\mu\left(H_{l}\right)-\mu\left(H_{r}\right)\right|<\varepsilon
$$

The threshold $\varepsilon$ is the threshold of the difference in intensity value between neighboring regions of an overlap candidate. In this research, the value of $\varepsilon$ is set as 0.082 (around 21 in grayscale), which is the optimal value obtained from the experiment. The small difference of intensity value means that the left and right neighboring regions have similar intensity, therefore it is probable that the candidate is located between two teeth, which means overlapping area.

\section{Experiments and analysis}

The dental panoramic radiograph images are obtained from hospital Rumah Sakit Gigi dan Mulut, Universitas Airlangga (RSGM UNAIR) [2]. The corresponding ground truth images of overlapping teeth area are created manually and confirmed by the radiological experts. This research used 24 different ROI images that contain overlapping teeth and 20 different ROI images that did not contain overlapping teeth, which then labeled as overlap and non-overlap class, respectively. Examples of ROI for each class can be seen in Fig. 5. The result of image enhancement and automatic marking of candidate points is shown in Fig. 6. The result of the mean-shift clustering and its combination with the marks obtained from the automatic marking process is shown in Fig. 7. The entire system is built by using MATLAB software.

\subsection{Accuracy of the overlapping area detection}

The proposed detection system was evaluated using 44 ROIs from dental panoramic radiograph images. The performance of the system on detecting image that contains overlapping teeth was measured by using accuracy, sensitivity, and specificity evaluation metrics. Accuracy measures the number of data that were correctly classified by the system. Sensitivity measures the number of overlapping data that were correctly classified by the system. Specificity measures the number of non-overlapping data that were correctly classified by the system. Let $T P$ be the overlapping data that were correctly classified, $T N$ be the non-overlapping data that were correctly classified, $F N$ be the overlapping data that were misclassified, and $F P$ be the non-overlapping data that were misclassified. The formula of

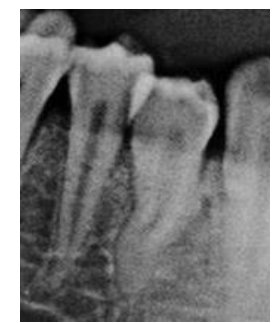

(a)

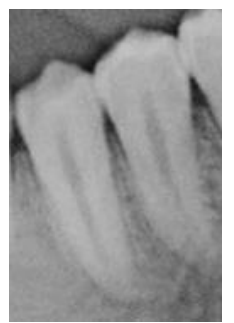

(b)
Figure. 5 Example of image data: (a) overlapping teeth class and (b) non-overlapping teeth class 


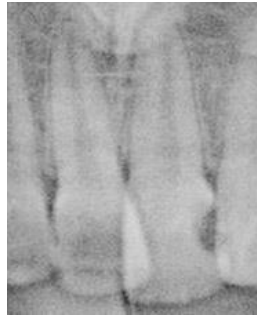

(a)

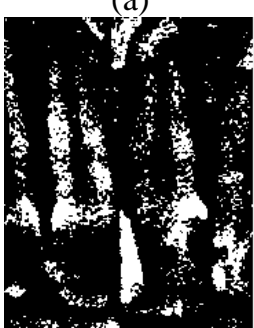

(c)

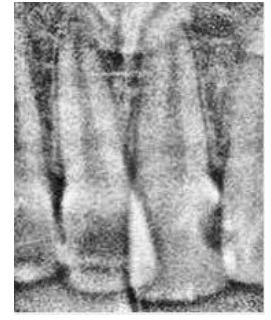

(b)

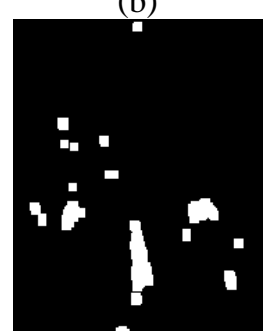

(d)
Figure. 6 The process of obtaining marking points: (a) input ROI image, (b) enhanced image, (c) result of the proposed thresholding, and (d) result of the morphological closing operation

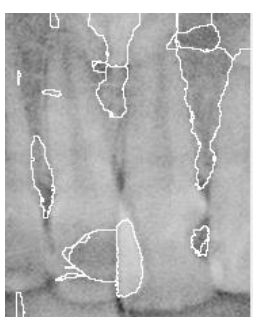

(a)

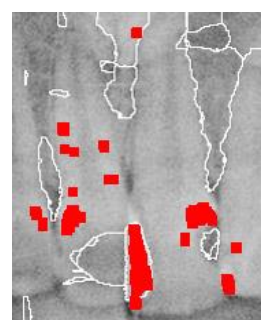

(b)

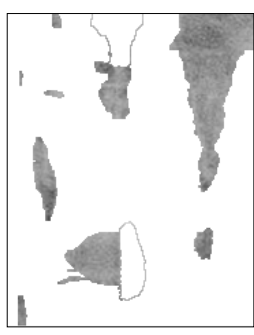

(c)
Figure. 7 The result of mean-shift image clustering combined with automatic marking: (a) image cluster by mean-shift algorithm, (b) marked points of high-intensity

pixel, and (c) clusters containing the marked points (indicated with white color)

calculating accuracy, sensitivity, and specificity is shown in Eq. (5) - Eq. (7), respectively.

$$
\begin{aligned}
& \text { Accuracy }=\frac{T P+T N}{T P+T N+F N+F P} \\
& \text { Sensitivity }=\frac{T P}{T P+F N} \\
& \text { Specificity }=\frac{T N}{T N+F P}
\end{aligned}
$$

The obtained accuracy, sensitivity, and specificity from all the test data are $75 \%, 66.67 \%$, and $85 \%$, respectively. The sensitivity value is not too high because the system sometimes makes mistakes on eliminating the overlap candidates when comparing the neighborhood intensity. The system eliminates the correct overlap candidates because the difference of its neighborhood intensity does not reach the minimum threshold $\varepsilon$, which means that the intensity of neighboring clusters of an overlapping teeth area is not quite similar. The specificity value is high because the proposed thresholding method in the automatic marking process gives an effective result on eliminating the non-overlapping area that has lower grayscale intensity than the overlapping area. The high accuracy value shows that the proposed system gives an effective detection of overlapping teeth on dental panoramic radiographs.

\subsection{Comparison of automatic marking methods}

The proposed method uses modified Otsu's thresholding for the automatic marking of coordinate points. The automatic marking process select pixels with high intensity as the marks of the location of the overlapping teeth area. The incorrect result of automatic marking may produce an error in the result of overlapping detection because the incorrectly marked area may lead to the nonoverlapping area incorrectly detected as overlapping area, and vice versa, in the selection process of overlap candidates.

In this experiment, we compared modified Otsu's thresholding as the automatic marking method with other automatic segmentation methods, which are Gaussian Mixture Model (GMM) [24] and Histogram Cluster Analysis (HCA) [25]. GMM and HCA methods are selected to provide equal comparison because the process of automatic marking uses automatic segmentation method that separates the grayscale intensity into four regions. Both HCA and GMM can separate the histogram of grayscale intensity into $k$ regions based on the graylevel value and its frequency. The number of $k$ in this experiment is four and the third threshold, that separates the third and fourth region, is selected to segment the high-intensity pixels as the marks of the overlapping area.

GMM models the histogram of grayscale intensity in the image as a mixture of several Gaussian distributions. The mean and standard deviation of each Gaussian distribution will be updated to maximize the likelihood of the graylevels assigned in each Gaussian distribution [24]. The result of GMM is $k$ Gaussian distributions that represent the distribution of grayscale intensity in the histogram, with $(k-1)$ thresholds that separate each of the Gaussian distribution. HCA assumes the gray-level in the histogram with a non-zero element as a cluster. The inter-class variance and intra-class variance of each cluster are then calculated to determine which clusters have a smaller distance. Hierarchical clustering is then carried out until there is only $k$ number of clusters left. 


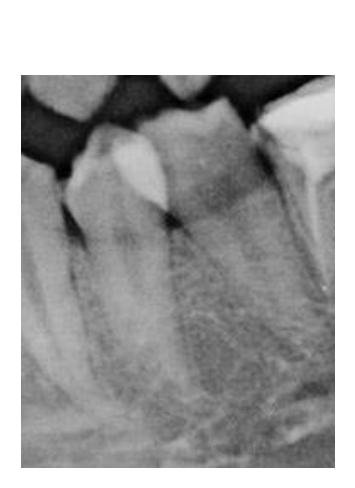

(a)

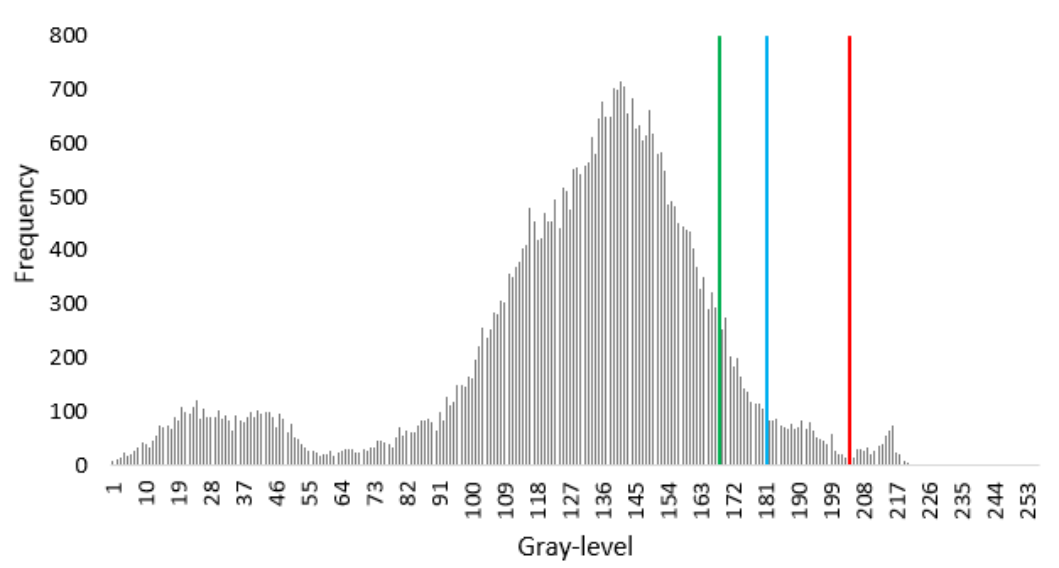

(b)

Figure. 8 (a) Example of an input image and (b) its histogram and the threshold of the proposed method (red line), GMM method (green line), and HCA method (blue line) for the automatic marking process

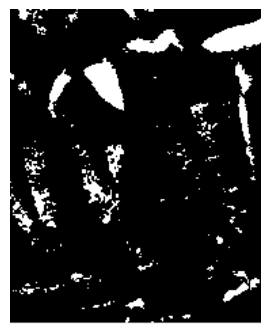

(a)

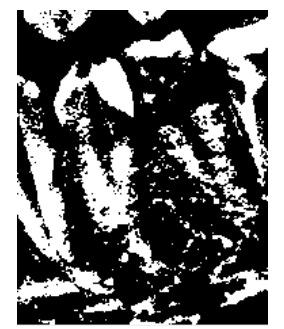

(b)

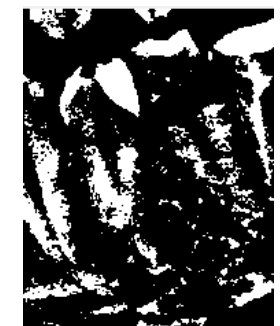

(c)
Figure. 9 The segmentation result by using the obtained threshold from: (a) the proposed method, (b) GMM method, and (c) HCA method

Table 1. Comparison of automatic marking methods

\begin{tabular}{|c|c|c|c|}
\hline Method & $\begin{array}{c}\text { Accuracy } \\
\%\end{array}$ & $\begin{array}{c}\text { Sensitivity } \\
\mathbf{\%}\end{array}$ & $\begin{array}{c}\text { Specificity } \\
\mathbf{\%}\end{array}$ \\
\hline Proposed & $\mathbf{7 5 . 0 0}$ & $\mathbf{6 6 . 6 7}$ & $\mathbf{8 5 . 0 0}$ \\
\hline $\begin{array}{c}\text { Gaussian } \\
\text { Mixture } \\
\text { Model }\end{array}$ & 59.09 & 62.50 & 55.00 \\
\hline $\begin{array}{c}\text { Histogram } \\
\text { Cluster } \\
\text { Analysis }\end{array}$ & 68.18 & $\mathbf{6 6 . 6 7}$ & 70.00 \\
\hline
\end{tabular}

The comparison results are shown in Tabel 1. By using GMM for automatic marking process, the system obtains the accuracy of $59.09 \%$, the sensitivity of $62.5 \%$, and the specificity of $55 \%$. By using HCA for automatic marking process, the system obtains the accuracy of $68.18 \%$, the sensitivity of $66.67 \%$, and the specificity of $70 \%$. Overall, the use of the proposed segmentation method provides better system performance than the use of the GMM and HCA methods. Fig. 8 shows the example of the obtained histogram threshold for automatic marking by using the proposed method (red line), GMM (green line), and HCA (blue line). The segmentation results of those three methods, which then will be further processed as the marking of overlapping teeth area, are shown in Fig. 9. It shows that the obtained threshold of the HCA method is quite similar with the obtained threshold of the proposed method, therefore the use of HCA method gives a more similar performance with the proposed method than the use of GMM method. It also shows that the proposed method is more effective in selecting the high-intensity pixels, which contains the overlapping area, than the GMM and HCA method.

\subsection{Error measurement of the segmented overlapping area}

To measure the performance of the segmentation result of the overlapping area, a comparison between the manually described overlapping area (ground truth) and the segmentation results of the system is done. The results of the system on segmenting the overlapping area are measured by using Misclassification Error (ME) value. Let $O_{g}$ and $B_{g}$ be the pixels of the overlapping area and background area, respectively, on the ground truth image. Let $O_{r}$ and $B_{r}$ be the pixels of the overlapping area and the background area, respectively, on the segmentation results of the system. The ME calculates the ratio between the number of misclassified pixels and the number of pixels in the image, according to Eq. (8). ME measurement only calculated on 24 test images that contain the overlapping teeth area. The average ME value of the proposed method is $0.31 \%$. The low $\mathrm{ME}$ value shows that the segmentation result of the overlapping teeth area in the proposed system is accurate. 


$$
M E=1-\frac{\left|o_{g} \cap O_{r}\right|+\left|B_{g} \cap B_{r}\right|}{\left|o_{g}\right|+\left|B_{g}\right|}
$$

\subsection{Comparison with segmentation methods}

semi-automatic

The methods for automatic detection of overlapping objects as mentioned in Section 2 usually can only be used on certain types of images because it utilizes the features that exist only in that image. On the other hand, other researches related to the detection of overlapping teeth on dental panoramic images have not yet been conducted. Therefore, because it is not possible to compare the results of the overlap area segmentation with automatic segmentation method, this experiment will compare segmentation results of the overlapping teeth area between the proposed method with the semi-automatic segmentation methods. Semi-automatic segmentation method needs some information provided manually by the user to conduct the segmentation process.

In this experiment, we compare the proposed method with the interactive image segmentation method proposed in [26] and distance regularized level set evolution (DRLSE) [27]. The interactive image segmentation method uses the mean-shift algorithm to divide the image into regions. Then the user marks several regions as the representative of the background cluster and the object cluster (overlap area). Region merging is then conducted to merge the remaining regions into those two clusters. The DRLSE method needs the user to provide an initialization area which located in the object (overlap) area. The initialization area will expand according to the energy functions and include the neighborhood areas that meet the criteria as part of the object.

Figs. 10-14 shows the example of the input images, ground truth images, the segmentation results of the proposed method, the segmentation results of the interactive image segmentation method, and the segmentation results of the DRLSE method, respectively. The average $\mathrm{ME}$ value of interactive image segmentation is $0.24 \%$. The average ME value of DRLSE method is $0.32 \%$. The segmentation error of the proposed method is higher than the segmentation error of the interactive image segmentation method because sometimes the result of the image clustering process is inaccurate and the automatic marking process fails to marks several regions that are included in the overlapping area, such as in Fig. 12 (a).

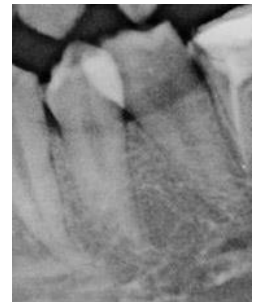

(a)

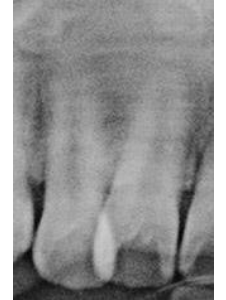

(b)

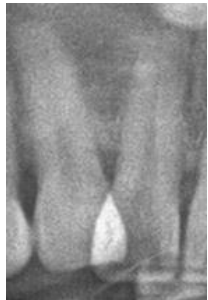

(c)
Figure. 10 Example of input images

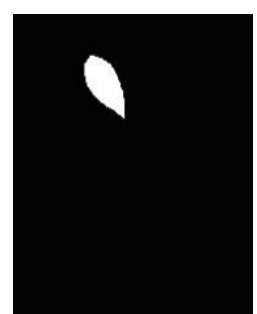

(a)

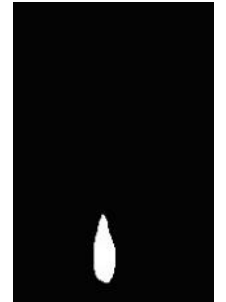

(b)

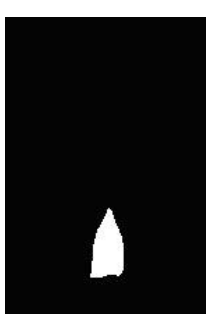

(c)
Figure. 11 Example of ground truth images

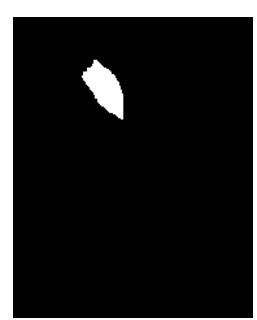

(a)

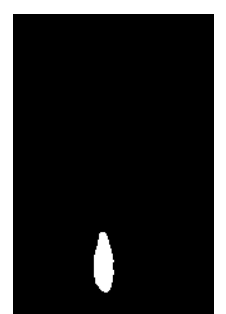

(b)

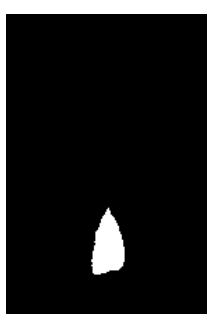

(c)
Figure. 12 Segmentation results by using the proposed method where: (a) $\mathrm{ME}=0.30 \%$, (b) $\mathrm{ME}=0.26 \%$, and (c) $\mathrm{ME}=0.31 \%$

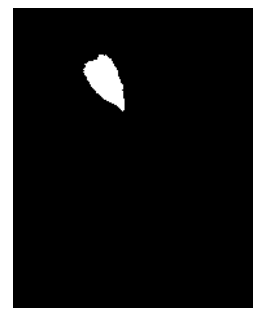

(a)

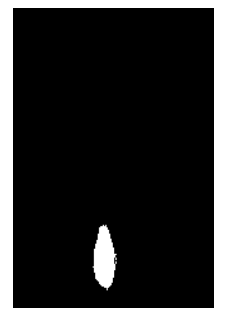

(b)

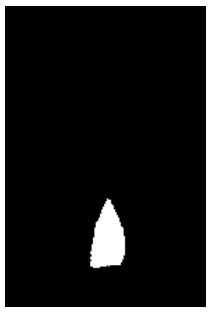

(c)
Figure: 13 Segmentation results by using interactive image segmentation where: (a) $\mathrm{ME}=0.28 \%$, (b) $\mathrm{ME}=0.17 \%$, and (c) $\mathrm{ME}=0.20 \%$

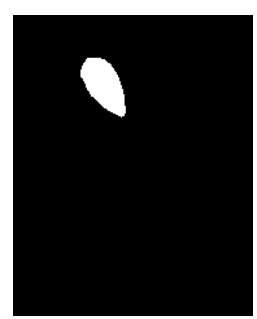

(a)

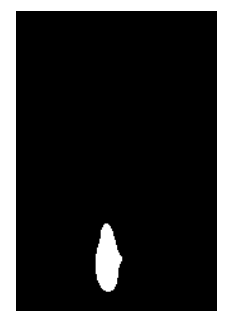

(b)

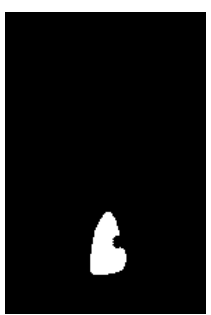

(c)
Figure. 14 Segmentation results by using DRLSE method where: (a) $\mathrm{ME}=0.29 \%$, (b) $\mathrm{ME}=0.29 \%$, and (c) $\mathrm{ME}=$ $0.59 \%$ 
The DRLSE method gives a little higher error than the proposed method because dental panoramic radiographs have low contrast, therefore the level set method can include the background area as the object, such as in Fig. 14(b). In Fig. 14(c), the error of DRLSE method occurred because the method detects different contour in the object area which is dismissed as the object. From this experiment, it can be concluded that the proposed method can give segmentation result of overlapping teeth area as good as the semi-automatic image segmentation methods. However, the semi-automatic image segmentation methods are not recommended on task that required a lot of data with a high variation because the marking or initialization process is exhaustive.

\section{Conclusion}

A new strategy to automatically detect an overlapping teeth area on dental panoramic radiograph was presented in this research. Based on the experimental results, the proposed thresholding method, which is conditioned according to the characteristics of the overlapping area, is able to obtain the overlapping area. A morphological closing operation can help eliminate the irrelevant small parts of the thresholding result and leave large objects that are used as marking points. The meanshift clustering is used to separate the image into clusters. By combining the mean-shift clustering result with the marking points, the candidates of the overlapping area can be obtained. The overlapping teeth area is detected by eliminating the overlap candidates according to its orientation and the similarity of neighborhood intensity.

The experiment is performed on 44 dental panoramic radiographs with 24 overlapping teeth images and 20 non-overlapping teeth images. The experimental results show that our proposed strategy can detect overlapping teeth on the dental panoramic radiograph with accuracy, sensitivity, and specificity of $75 \%, 66.67 \%$, and $85 \%$, respectively. The evaluation conducted on 24 overlapping teeth images shows that the segmentation results of overlapping teeth area have an average misclassification error of $0.31 \%$. For future work, this proposed strategy can be further developed for overlapping teeth separation and single tooth segmentation.

\section{Acknowledgments}

This work is supported by the Ministry of Research, Technology, and Higher Education,
Indonesia, with grant number 5/E1/KP.PTNBH/2019.

\section{References}

[1] S. Yuheng and Y. Hao, "Image Segmentation Algorithms Overview", Architecture and Algorithms for Digital Image Processing II, Vol. 0534, pp. 172, 2012.

[2] R. Indraswari, A. Z. Arifin, D. A. Navastara, and N. Jawas, "Teeth segmentation on dental panoramic radiographs using decimation-free directional filter bank thresholding and multistage adaptive thresholding", In: Proc. of 2015 International Conf. on Information and Communication Technology and System (ICTS 2015), pp. 49-54, 2016.

[3] S. D. Na, G. Lee, J. H. Lee, and M. N. Kim, "Individual tooth region segmentation using modified watershed algorithm with morphological characteristic", Bio-medical Materials and Engineering, Vol. 24, No. 6, pp. 3303-3309, 2014.

[4] A. Poonsri, N. Aimjirakul, T. Charoenpong, and C. Sukjamsri, "Teeth segmentation from dental x-ray image by template matching", In: Proc. of 2016 9th Biomedical Engineering International Conf. (BMEiCON), pp. 1-4, 2016.

[5] P. L. Lin, P. Y. Huang, P. W. Huang, H. C. Hsu, and C. C. Chen, "Teeth segmentation of dental periapical radiographs based on local singularity analysis", Computer Methods and Programs in Biomedicine, Vol. 113, No. 2, pp. 433-445, 2014.

[6] N. S. Ahmad, Z. M. Zaki, and W. Ismail, "Region of adaptive threshold segmentation between mean, median and otsu threshold for dental age assessment", In: Proc. of 2014 International Conf. on Computer, Communications, and Control Technology (I4CT), pp. 353-356, 2014.

[7] R. Kurniawan, I. Muhimmah, A. Kurniawardhani, and I. Indrayanti, "Segmentation of Overlapping Cervical Cells in Normal Pap Smear Images Using DistanceMetric and Morphological Operation", CommIT (Communication and Information Technology) Journal, Vol. 11, No. 1, pp. 25-31, 2017.

[8] Z. Wang, K. Wang, F. Yang, S. Pan, and Y. Han, "Image segmentation of overlapping leaves based on Chan-Vese model and Sobel operator", Information Processing in Agriculture, Vol. 5, No. 1, pp. 1-10, 2018.

[9] M. R. M. Razali, N. S. Ahmad, R. Hassan, Z. 
M. Zaki, and W. Ismail, "Sobel And Canny Edges Segmentations For The Dental Age Assessment", In: Proc. of International Conference on Computer Assisted System in Health, pp. 62-66, 2014.

[10] P. L. Lin, Y. H. Lai, and P. W. Huang, "An effective classification and numbering system for dental bitewing radiographs using teeth region and contour information", Pattern Recognition, Vol. 43, No. 4, pp. 1380-1392, 2010.

[11] M. Abdel-Mottaleb, O. Nomir, D. E. Nassar, G. Fahmy, and H. H. Ammar, "Challenges of Developing an Automated Dental Identification System", In: Proc. of 2003 46th Midwest Symposium on Circuits and Systems, pp. 411414, 2003.

[12] T. L. Mahyari and R. M. Dansereau, "Random walks for image segmentation containing translucent overlapped objects", In: Proc. of IEEE Global Conf. on Signal and Information Processing, pp. 46-50, 2017.

[13]D. Riana, A. N. Hidayanto, D. H. Widyantoro, T. L. R. Mengko, and O. Kalsoem, "Segmentation of overlapping cytoplasm and overlapped areas in Pap smear images", In: Proc. of 2017 8th International Conf. on Information, Intelligence, Systems \& Applications (IISA 2017), Vol. 2018, pp. 1-5, 2017.

[14] M. Yadollahi, A. Procházka, M. Kašparová, and O. Vyšata, "Separation of overlapping dental objects using normal vectors to image region boundaries", In: Proc. of 2015 International Workshop on Computational Intelligence for Multimedia Understanding (IWCIM 2015), pp. 3-6, 2015.

[15]K. Zuiderveld, Contrast Limited Adaptive Histogram Equalization. Academic Press, New York, 2013.

[16] R. Xia, P. Wang, W. Zhang, and Q. Xiong, "A novel overlapping mice macrophages images segmentation method", In: Proc. of 2009 International Conf. on Image Analysis and Signal Processing (IASP 2009), pp. 40-43, 2009.

[17] N. Otsu, "A Threshold Selection Method from Gray-Level Histograms", IEEE Transactions on Systems, Man, and Cybernetics, Vol. 9, No. 1, pp. 62-66, 1979.

[18] P. Salembier and J. Serra, "Flat Zones Filtering, Connected Operators, and Filters by Reconstruction", IEEE Transactions on Image Processing, Vol. 4, No. 8, pp. 1153-1160, 1995.
[19] K. Wisaeng, “Exudates Detection Using Morphology Mean Shift Algorithm in Retinal Images", IEEE Access, Vol. 7, pp. 1194611958, 2019.

[20] Y. Cheng, "Mean Shift, Mode Seeking, and Clustering", IEEE Transactions on Pattern Analysis and Machine Intelligence, Vol. 17, No. 8, pp. 790-799, 1995.

[21] D. Comaniciu and P. Meer, "Mean shift: A robust approach toward feature space analysis", IEEE Transactions on Pattern Analysis and Machine Intelligence, Vol. 24, No. 5, pp. 603619, 2002.

[22] "Edison software," [Online]. Available: http://www.caip.rutgers.edu/riul/research/code. html

[23] J. Ning, L. Zhang, D. Zhang, and C. Wu, "Interactive image segmentation by maximal similarity based region merging", Pattern Recognition, Vol. 43, No. 2, pp. 445-456, 2010.

[24] R. Indraswari, A. Z. Arifin, N. Suciati, E. R. Astuti, and T. Kurita, "Automatic Segmentation of Mandibular Cortical Bone on Cone-Beam CT Images Based on Histogram Thresholding and Polynomial Fitting", International Journal of Intelligent Engineering and Systems, Vol. 12, No. 4, pp. 130-141, 2019.

[25] A. Z. Arifin and A. Asano, "Image segmentation by histogram thresholding using hierarchical cluster analysis", Pattern Recognition Letters, Vol. 27, No. 13, pp. 15151521, 2006.

[26] A. Z. Arifin, R. Indraswari, N. Suciati, E. R. Astuti, and D. A. Navastara, "Region merging strategy using statistical analysis for interactive image segmentation on dental panoramic radiographs", International Review on Computers and Software, Vol. 12, No. 1, pp. 63-74, 2017.

[27] C. Li, C. Xu, C. Gui, and M. D. Fox, "Distance regularized level set evolution and its application to image segmentation", IEEE Transactions on Image Processing, Vol. 19, No. 12, pp. 3243-3254, 2010. 